\title{
The presence of fever in adults with influenza and other viral respiratory infections
}

\author{
A. A. CHUGHTAI ${ }^{1 *}$, Q. WANG ${ }^{2}$, T. C. DUNG ${ }^{3}$ And C. R. MACINTYRE ${ }^{1,4}$ \\ ${ }^{1}$ School of Public Health and Community Medicine, UNSW Medicine, University of New South Wales, Sydney, \\ Australia \\ ${ }^{2}$ The Beijing Centre for Disease Prevention and Control, Beijing, China \\ ${ }^{3}$ National Institute of Hygiene and Epidemiology (NIHE), Vietnam \\ ${ }^{4}$ College of Public Service \& Community Solutions, Arizona State University, Phoenix, AZ, USA
}

Received 11 June 2016; Final revision 3 August 2016; Accepted 5 August 2016; first published online 3 October 2016

\section{SUMMARY}

We compared the rates of fever in adult subjects with laboratory-confirmed influenza and other respiratory viruses and examined the factors that predict fever in adults. Symptom data on 158 healthcare workers (HCWs) with a laboratory-confirmed respiratory virus infection were collected using standardized data collection forms from three separate studies. Overall, the rate of fever in confirmed viral respiratory infections in adult HCWs was $23 \cdot 4 \%$ (37/158). Rates varied by virus: human rhinovirus $(25 \cdot 3 \%, 19 / 75)$, influenza A virus $(30 \%, 3 / 10)$, coronavirus $(28 \cdot 6 \%, 2 / 7)$, human metapneumovirus $(28 \cdot 6 \%, 2 / 7)$, respiratory syncytial virus $(14 \cdot 3 \%, 4 / 28)$ and parainfluenza virus $(8 \cdot 3 \%, 1 / 12)$. Smoking [relative risk (RR) $4 \cdot 65,95 \%$ confidence interval (CI) $1 \cdot 33-16 \cdot 25$ ] and coinfection with two or more viruses (RR 4.19, 95\% CI 1.21-14.52) were significant predictors of fever. Fever is less common in adults with confirmed viral respiratory infections, including influenza, than described in children. More than $75 \%$ of adults with a viral respiratory infection do not have fever, which is an important finding for clinical triage of adult patients with respiratory infections. The accepted definition of 'influenza-like illness' includes fever and may be insensitive for surveillance when high case-finding is required. A more sensitive case definition could be used to identify adult cases, particularly in event of an emerging viral infection.

Key words: Fever, influenza-like illness, viral infection.

\section{INTRODUCTION}

Respiratory infections are common and one of the leading causes of morbidity and mortality, particularly in the extremes of age [1-3]. Influenza A and B, human rhinoviruses (HRV), respiratory syncytial virus (RSV), adenoviruses (ADV) and parainfluenza virus (PIV) are common respiratory viruses in adults and children

\footnotetext{
* Author for correspondence: Dr A. A. Chughtai, Level 2, Samuels Building, School of Public Health \& Community Medicine, UNSW Medicine, University of New South Wales, Sydney 2052, Australia. (Email: abrar.chughtai@unsw.edu.au)
}

[1-5]. Of respiratory infections, influenza is the most well studied viral infection, and is commonly reported (around 50\%) as the cause of epidemics of respiratory infection, including nosocomial outbreaks [6]. Influenza virus is commonly isolated from febrile paediatric and elderly patients presenting with influenza-like illness (ILI) and acute respiratory illness (ARI) symptoms [1]. The accepted clinical case definition of ILI includes fever, which may be suitable for identifying paediatric cases, but less so for adults.

Fever is thought of as the most common presenting symptom of influenza in hospital emergency departments; however, the presence of fever depends on the age of 
person and the type of virus [7-10]. It is known that fever is less common in adults than children with influenza, and that adults may have atypical presentations $[5,6,11]$. In a matched case-control study in Finland, 317 laboratoryconfirmed influenza cases and 353 controls with respiratory symptoms were recruited in children aged $\leqslant 13$ years. Fever was present in $89 \cdot 8 \%$ (317/353) and 35.7\% (126/353) of cases and controls, respectively [12]. In contrast to this, fever is not a common presentation in adults with laboratory-confirmed influenza. Monto et al. [13] examined clinical trial data of 3744 adult ILI cases (defined as body temperature $\geqslant 37.8^{\circ} \mathrm{C}$ or patients subjective feeling of feverishness) and of those $2470(66 \%)$ had laboratory-confirmed influenza. Fever $\left(\geqslant 37.8^{\circ} \mathrm{C}\right)$ was reported in $68 \%$ of laboratory-confirmed influenza cases, compared to $40 \%$ other ILI cases [13]. During a randomized clinical trial (RCT) around the efficacy of facemask and hand hygiene in the household setting, 44\% (15/34) of secondary cases with influenza A and 32\% (8/ $25)$ of cases with influenza B had fever history [14]. The rate of fever was $66 \%$ (137/207) in hospitalized influenza cases in a US study [15]. Another US study showed that less than half $(42 \cdot 4 \%)$ of healthcare workers $(\mathrm{HCWs})$ with laboratory-confirmed influenza presented with fever [5]. Fever is a less common presenting symptom in elderly people which may lead to diagnostic and treatment delays [16]. In patients admitted with myocardial infarction, 9\% had unrecognized and undiagnosed influenza on testing at admission, highlighting the low level of clinical suspicion of influenza [17].

The rate of fever also varies between influenza strains, being more common in influenza A strains than $\mathrm{B}$, and higher in H3N2 [7-10, 18]. Fever is a commonly reported symptom during influenza outbreaks and pandemics due to novel and more virulent nature of strains. In China $67 \cdot 4 \%$ of the patients infected by influenza $\mathrm{A}(\mathrm{H} 1 \mathrm{~N} 1) \mathrm{pdm} 09$ had fever [19]. In another study in Beijing 465 suspected ILI cases were tested and of those $318(68 \%)$ were positive for influenza virus (pandemic H1N1-165 and seasonal influenza H3N2-153) and all had history of fever [20].

The aim of this study was to compare the rates of fever in adult subjects with confirmed influenza and other respiratory virus infections and examine predictors of fever.

\section{METHODS}

We analysed a dataset of laboratory-confirmed viral respiratory infections collected from three clinical trials of $\mathrm{HCWs}$ where active surveillance for respiratory viral illness was conducted in prospective follow up [21-23]. The same methods, data collection forms and outcome measures, were used across the three studies, allowing the data to be pooled [21-23]. Two studies were conducted in Beijing China: trial 1 (2008/2009) and trial $2(2009 / 2010)$ and another study (trial 3) was conducted in Hanoi, Vietnam in 2010/2011 [21-23]. In all clinical trials, participants were asked to complete diary cards on a daily basis to collect information on number of working hours, patients seen, mask use hours, high-risk procedures performed and appearance of respiratory symptoms. Thermometers were given the participants to measure their temperature daily and at symptom onset. Symptomatic cases were asked to complete sick patient follow-up forms and detailed information was collected on the following symptoms: chill or fever, cough, congestion, runny nose, sore throat, sneezes, lethargy, loss of appetite, abdominal pain, muscle or joint aches. Swabs of both tonsils and the posterior pharyngeal wall were collected on the day of reporting.

In all RCTs, fever was defined as having body temperature $\geqslant 38^{\circ} \mathrm{C}$. Clinical respiratory illness (CRI) and ILI were in the primary outcomes in three clinical trials. CRI was defined as two or more respiratory symptoms or one respiratory symptom and a systemic symptom and ILI was defined as fever $\geqslant 38^{\circ} \mathrm{C}$ plus one respiratory symptom [21-23].

\section{Analysis}

We analysed data from all subjects with a positive isolation of a respiratory virus by multiplex polymerase chain reaction (PCR). Descriptive analysis was conducted for rates of fever by virus type. A logistic regression analysis was used to determine the predictors of fever. A multivariable log binomial model was fitted, using a generalized linear model to estimate relative risk (RR). All variables were included in initial model. In the final model, we included only those variables that were significant $(P<0 \cdot 25)$ in initial analysis. A backward elimination method was used to remove the variables that did not have any confounding effect, i.e. could not make meaningful change $( \pm 10 \%)$ in the RR of the comparison arm. Finally we estimated the rates of CRI and ILI in the laboratory-confirmed viral respiratory infections and laboratory-confirmed influenza infections. The data was analysed using SAS v. 9.4 (SAS Institute Inc., USA). 
Table 1. Demographic characteristics of cases in three clinical trials

\begin{tabular}{lll}
\hline \hline Variable & No. & Percent \\
\hline Country & & \\
$\quad$ China (trials 1 and 2) & 90 & 57 \\
$\quad$ Vietnam (trial 3) & 68 & 43 \\
Profession & & \\
$\quad$ Doctors & 56 & $35 \cdot 4$ \\
$\quad$ Nurses & 102 & $64 \cdot 6$ \\
Age, years, mean (s.D.) & & $32 \cdot 8( \pm 9 \cdot 14)$ \\
Gender & & \\
$\quad$ Male & 21 & $13 \cdot 3$ \\
$\quad$ Female & 137 & $86 \cdot 7$ \\
Smoking status & & \\
$\quad$ Current/ex-smoker & 12 & $7 \cdot 6$ \\
$\quad$ No & 146 & $92 \cdot 4$ \\
Influenza vaccine & & \\
$\quad$ Yes & 22 & $13 \cdot 9$ \\
$\quad$ No & 136 & $86 \cdot 1$ \\
Virus type & & \\
$\quad$ Co-infection & 15 & $9 \cdot 5$ \\
$\quad$ Human rhinovirus & 75 & $47 \cdot 5$ \\
$\quad$ Respiratory syncytial virus & 28 & $17 \cdot 7$ \\
$\quad$ Influenza A and B viruses & 13 & $8 \cdot 2$ \\
$\quad$ Other* & 27 & $17 \cdot 1$ \\
Fever & & \\
$\quad$ Yes & 37 & $23 \cdot 4$ \\
$\quad$ No & 121 & $76 \cdot 6$ \\
\hline \hline
\end{tabular}

* Other includes: parainfluenza virus $(n=12)$, human metapneumovirus $(n=7)$, coronavirus $(n=7)$ and adenovirus $(n=1)$.

\section{Ethical approval}

Ethical approval of two clinical trials (China trials 20082009 and 2009-2010) were obtained from the Institutional Review Board and Human Research Ethics Committee of the Beijing Center for Disease Prevention and Control. For the Vietnam trial ethical approval was obtained from National Institute for Hygiene and Epidemiology (NIHE) (approval no. 05 IRB) and the Human Research Ethics Committee of the University of New South Wales (UNSW), Australia (HREC approval no. 10306). All participants provided written informed consent prior to commencing the trials.

\section{RESULTS}

The demographic characteristics of 158 cases with laboratory-confirmed viral infections are presented in Table 1 . Ninety $(57 \%)$ cases were from China and $68(43 \%)$ were from Vietnam. The mean age of HCWs was $32 \cdot 8$ years and most participants were nurses $(65 \%)$ and female $(87 \%)$. Most cases were non-
Table 2. Rate of fever in respiratory infections in the pooled dataset of three clinical trials

\begin{tabular}{|c|c|c|c|}
\hline Viruses isolated & $\begin{array}{l}\text { No. of } \\
\text { cases }\end{array}$ & $\begin{array}{l}\text { Case } \\
\text { with } \\
\text { fever }\end{array}$ & $\begin{array}{l}\text { Rate of } \\
\text { fever }(\%)\end{array}$ \\
\hline Adenovirus & 1 & 0 & 0 \\
\hline Coronavirus & 7 & 2 & $28 \cdot 6$ \\
\hline Influenza $A$ virus & 10 & 3 & $30 \cdot 0$ \\
\hline $\begin{array}{l}\text { Influenza A virus/human } \\
\text { rhinovirus }\end{array}$ & 3 & 3 & 100 \\
\hline Influenza B virus & 3 & 0 & 0 \\
\hline $\begin{array}{l}\text { Influenza B virus/human } \\
\text { rhinovirus }\end{array}$ & 2 & 2 & 100 \\
\hline $\begin{array}{l}\text { Influenza B virus/ } \\
\text { parainfluenza }\end{array}$ & 2 & 0 & 0 \\
\hline Human rhinovirus & 75 & 19 & $25 \cdot 3$ \\
\hline Parainfluenza virus & 12 & 1 & $8 \cdot 3$ \\
\hline $\begin{array}{l}\text { Parainfluenza virus } \\
\text { /coronavirus }\end{array}$ & 1 & 0 & 0 \\
\hline $\begin{array}{l}\text { Parainfluenza/influenza } \\
\text { A virus }\end{array}$ & 1 & 0 & 0 \\
\hline Respiratory syncytial virus & 28 & 4 & $14 \cdot 3$ \\
\hline $\begin{array}{l}\text { Respiratory syncytial virus/ } \\
\text { influenza A virus }\end{array}$ & 1 & 0 & 0 \\
\hline $\begin{array}{l}\text { Respiratory syncytial virus/ } \\
\text { human rhinovirus }\end{array}$ & 3 & 0 & 0 \\
\hline $\begin{array}{l}\text { Respiratory syncytial virus/ } \\
\text { parainfluenza }\end{array}$ & 1 & 1 & 100 \\
\hline Human metapneumovirus & 7 & 2 & $28 \cdot 6$ \\
\hline $\begin{array}{l}\text { Human metapneumovirus/ } \\
\text { human rhinovirus }\end{array}$ & 1 & 0 & 0 \\
\hline Total & 158 & 37 & $23 \cdot 4$ \\
\hline All influenza* & 22 & 8 & $36 \cdot 4$ \\
\hline
\end{tabular}

* Includes co-infection with other viruses.

smokers $(92 \%)$ and had not received influenza vaccine $(86 \%)$. Viruses isolated included rhinovirus $(n=75$, $47 \%)$, RSV $(n=28,18 \%)$, influenza $(n=13,8 \%)$, PIV $(n=12,8 \%)$, human metapneumovirus (hMPV; $n=7$, $4 \%)$, coronavirus $(n=7,4 \%)$ and $\operatorname{ADV}(n=1,1 \%)$. More than one virus was isolated in 15 cases $(9 \cdot 5 \%)$, including nine cases with influenza co-infection. Fever was documented in $23 \cdot 4 \%$ cases $(37 / 158)$ with a positive laboratory viral diagnosis.

Table 2 details rates of fever $\left(\geqslant 38^{\circ} \mathrm{C}\right)$ associated with individual viral respiratory infections. HRV was the most common infection and 25.3\% (19/75) of these had a fever. In 28 cases of RSV, four (14.3\%) had fever; $8 \cdot 3 \%(1 / 12)$ of PIV and 30\% (3/10) of influenza A cases had fever. Seven cases of coronavirus and hMPV each were confirmed and of those two (28.6\%) had fever. When cases with influenza and a co-infection were included, $36 \cdot 4 \%(8 / 22)$ had fever. 
Table 3. Predictors of fever in cases with viral respiratory infections

\begin{tabular}{|c|c|c|c|c|}
\hline Variable & $\begin{array}{l}\text { Cases with } \\
\text { fever }(n / N)\end{array}$ & Rate (\%) & $\begin{array}{l}\text { Univariate analysis } \\
(\mathrm{RR}, 95 \% \mathrm{CI})\end{array}$ & $\begin{array}{l}\text { Multivariate analysis } \\
(\mathrm{RR}, 95 \% \mathrm{CI})\end{array}$ \\
\hline \multicolumn{5}{|l|}{ Country } \\
\hline China (trials 1 and 2) & $15 / 90$ & $16 \cdot 7$ & Ref. & Ref. \\
\hline Vietnam (trial 3) & $22 / 68$ & $32 \cdot 4$ & $2 \cdot 39(1 \cdot 13-5 \cdot 07)$ & $2.99(1 \cdot 24-7 \cdot 20)$ \\
\hline \multicolumn{5}{|l|}{ Profession } \\
\hline Doctors & $12 / 56$ & $21 \cdot 4$ & $0 \cdot 84(0 \cdot 38-1 \cdot 84)$ & \\
\hline Nurses & $25 / 102$ & $24 \cdot 5$ & Ref. & \\
\hline Age & & & $0 \cdot 96(0 \cdot 92-1 \cdot 01)$ & \\
\hline \multicolumn{5}{|l|}{ Gender } \\
\hline Male & $9 / 21$ & $42 \cdot 9$ & $2 \cdot 92(1 \cdot 12-7 \cdot 62)$ & \\
\hline Female & $28 / 137$ & $20 \cdot 4$ & Ref. & \\
\hline \multicolumn{5}{|l|}{ Smoking status } \\
\hline Current/ex-smoker & $7 / 12$ & $58 \cdot 3$ & $5 \cdot 41(1 \cdot 60-18 \cdot 26)$ & $4 \cdot 65(1 \cdot 33-16 \cdot 25)$ \\
\hline No & $30 / 146$ & $20 \cdot 5$ & Ref. & Ref. \\
\hline \multicolumn{5}{|l|}{ Influenza vaccine } \\
\hline Yes & $6 / 22$ & $27 \cdot 3$ & $1 \cdot 27(0 \cdot 46-3 \cdot 52)$ & \\
\hline No & $31 / 136$ & $22 \cdot 8$ & Ref. & \\
\hline \multicolumn{5}{|l|}{ Type of virus } \\
\hline Influenza virus & $3 / 13$ & $23 \cdot 1$ & $1 \cdot 09(0 \cdot 28-4 \cdot 24)$ & $1 \cdot 89(0 \cdot 43-8 \cdot 31)$ \\
\hline All other viruses* & $28 / 130$ & $21 \cdot 5$ & Ref. & Ref. \\
\hline Co-infection & $6 / 15$ & 40 & $2 \cdot 43(0 \cdot 80-7 \cdot 40)$ & $4 \cdot 19(1 \cdot 21-14 \cdot 52)$ \\
\hline
\end{tabular}

RR, Relative risk; CI, confidence interval.

* Includes human rhinovirus $(n=75)$, respiratory syncytial virus $(n=28)$, parainfluenza virus $(n=12)$, human metapneumovirus $(n=7)$, coronavirus $(n=7)$ and adenovirus $(n=1)$.

In univariate analysis, country, gender and smoking were significant predictors of fever. Country and smoking remained significant predictors in multivariate analysis while gender became non-significant. Fever rate was significantly higher in HCWs in Vietnam compared to HCWs in China [RR 2.99, 95\% confidence interval (CI) 1.24-7.20]. Smokers were around five times more likely to have fever compared to non-smokers (RR 4.65, 95\% CI 1·33-16.25). Virus type was not associated with fever in univariate analysis; however, after adjusting for other variables, rates of fever were significantly higher in HCWs co-infected with more than one virus compared to all other viruses excluding influenza (RR 4·19, 95\% CI 1·21-14.52) (Table 3).

CRI symptoms were present in $84.8 \%$ (137/158) of HCWs with laboratory-confirmed viral infections and $90 \cdot 9 \%$ (20/22) laboratory-confirmed influenza infections. The corresponding rates of ILI in the two groups were $9 \cdot 5 \%(15 / 158)$ and $13 \cdot 6 \%(3 / 22)$, respectively.

\section{DISCUSSION}

We have shown, using prospectively collected data, that the rate of fever in adults with confirmed viral respiratory infections is much lower than described in children
$[1,9]$. The standard clinical case definition of ILI requires fever to be present - the majority of influenza cases in this series would have been missed using the ILI definition. This has implications for effective triage, early antiviral treatment and preventive measures for adults with influenza, particularly during outbreaks and pandemic situations. For other respiratory infections, clinical case definitions need to be more sensitive, or $>75 \%$ of cases will be missed. The main implication for future surveillance, measurements and research studies is that the ILI case definition in adults may be highly insensitive. For some types of surveillance systems, this may not be an issue, but for diagnostic screening in event of an emerging viral infection (such as for triage and implementation of infection control protocols) [24, 25], a more sensitive case definition is needed.

Rates of fever in influenza and other viral respiratory infections in this study were lower compared to other studies which report fever in around $50-70 \%$ adult cases [1, 5, 13, 15]. However, this variation may be due to different study base, case definition and viral strains, as well as the prospective measurement of incident infections. Many research studies use fever as an inclusion criterion for laboratory testing $[13,26]$. While this may be suitable for studies in 
children, it is not adequately sensitive for studies of adults, as we have shown the majority of confirmed cases will be missed. The cut-off point for fever could be another factor in sensitivity. Some studies have set lower cut-off points for fever, and report higher rates of fever in laboratory-confirmed influenza cases [13, 27]. Carrat et al. collected data of cases presented in 35 general practices in France and collected nasal swabs from suspected influenza cases and defined fever as $\geqslant 37 \cdot 8^{\circ} \mathrm{C}$. They found fever in influenza $\mathrm{A}(\mathrm{H} 3 \mathrm{~N} 2)$, influenza $\mathrm{A}(\mathrm{H} 1 \mathrm{~N} 1)$ and influenza negative cases in $95 \cdot 2 \%, 77 \cdot 5 \%$ and $72 \cdot 7 \%$, respectively. Applying a cut-off of $\geqslant 38.2{ }^{\circ} \mathrm{C}$, the corresponding rates are $82 \cdot 2 \%, 59 \cdot 3 \%$ and $43 \cdot 9 \%$ [27]. Symptoms of feverishness (subjective feeling of fever) are included in ILI definitions in some cases [13].

Previous studies report high rates of fever in children compared to the adults [11]. Low rates of fever in adults may also be due to protection via cross-reactive antibodies due to age-dependent differences in the immunity $[7,28]$. Continued exposure to influenza throughout life may result in a broader protection with age. Infection may provoke a stronger immune response in children with minimal to no exposure history compared to adults. Therefore influenza infection history might help explain potential differences in clinical symptom severity (and presence of fever) between children and adults.

A recent study reported high rates of influenza and other respiratory virus in afebrile $\mathrm{HCWs}$ with only respiratory symptoms [5]. Of 22 laboratory-confirmed influenza cases in this study, only three $(13 \cdot 6 \%)$ had ILI symptoms, which is very low compared to other studies. In a prospective influenza surveillance study, ILI symptoms were present in $48 \%$ of adults and $61 \%$ of children with laboratory-confirmed influenza virus [1]. CRI symptoms were present in $90.9 \%(20 / 22)$ of laboratory-confirmed influenza cases in this study.

A highly sensitive definition of influenza may be required to diagnose most of adult influenza cases in the clinical setting to ensure rapid treatment and isolation, and prevention of nosocomial transmission. Inclusion of ILI cases may overestimate the proportion of febrile cases in influenza surveillance given fever is included in the definition. Pre-symptomatic and asymptomatic influenza cases will also be missed, although infectivity and transmissibility of these cases is yet to be proven [29]. Longitudinal studies, where all participants are tested, provide similar estimates around rates of fever as in our study [14]. We propose a more sensitive clinical case definition without fever as a requisite criterion.
Clinical signs and symptoms are less studied for other viral respiratory infections, but available evidence suggests that other respiratory viruses are associated with a lower rate of fever compared to influenza [5, 30-33]. Putto and colleagues [30] examined the clinical records of 258 children ( $>3$ months) in a large hospital in Finland, including ADV (25 cases), influenza A and B (74 cases), PIV (99 cases) and RSV (60 cases). Fever $\left(\geqslant 39 \cdot 0{ }^{\circ} \mathrm{C}\right)$ was recorded in $68 \%$ cases with ADV, $84 \%$ influenza A virus, $65 \%$ influenza B, $41 \%$ PIV-1, $50 \%$ PIV-2, $47 \%$ PIV-3, and 52\% RSV. Van den Hoogen and colleagues estimated the prevalence and clinical symptoms of hMPV infection, in The Netherlands and fever was reported in $61 \%$ of the hMPV-positive cases [31]. In Hong Kong, hMPV was found in 5.5\% (32/587) of children admitted in hospitals and all had fever [32]. Manoha et al. examined nasal wash specimens from 931 hospitalized children and found hMPV $(6 \%)$, RSV $(28 \cdot 5 \%)$, rhinoviruses $(18 \cdot 3 \%)$, influenza A $(6 \%)$, PIV-1 $(0 \cdot 2 \%)$ and PIV-3 $(0 \cdot 3 \%)$. Fever was reported in $39 \cdot 2 \%$ cases with hMPV, $37 \cdot 8 \%$ cases with RSV and $30 \cdot 2 \%$ with rhinovirus [33]. Of the 210 elderly patients with influenza and 145 with RSV, fever was reported in $65 \%$ and $50 \%$, respectively [3]. A US study also reported low rates of fever in $\mathrm{HCW}$ s infected with coronavirus 229E (13.5\%), coronavirus HKU (11.4\%), coronavirus NL63 (31.3\%) and RSV (12.9\%) and all cases of hMPV were without fever [5]. Rate of fever for all other viruses (excluding influenza) was $21 \cdot 5 \%(28 / 130)$ in this study.

Co-infection with more than one virus was the strongest predictor of fever for adults with confirmed viral respiratory infections in the present study. Previous studies also show high rates of fever in cases with dual respiratory viral infections compared to single viral infection [34, 35]. Rates of hospitalization and ICU admission are also reported to be higher in cases with dual respiratory viral infections [36-38]. Increased severity of symptoms in co-infection cases might be due to an altered immune response [34]. Around $10 \%(15 / 158)$ of cases in our dataset were infected with more than one virus. Drews et al. reviewed the data of eight prospective epidemiological studies and reported the rate of co-infection was 5\% [37]. Studies in children generally report higher rates of co-infection cases $(17-20 \%)$ [34, 35, 38, 39]. Clinicians should consider the possibly of co-infection if a patient presents with fever; however, further epidemiological and clinical studies are required.

Smoking was also a significant predictor of fever in this study. Smoking increases the risk of viral and 
bacterial infections through changes in respiratory epithelial and altered immune response [40-43]. The risk of influenza also increases several times in smokers, compared to non-smokers [40]. Atypical clinical presentation of influenza and other respiratory infections in adults could be due to altered structural and immune response associated with active/passive smoking and other environmental hazards. The mechanism by which smoking increases the risk of fever is not clear. High rates of fever in smokers may also be due changes in immunoglobulin levels which could increase viral load. The severity of symptoms generally increases when high viral load is detected in the blood [44].

The difference in fever rates between China and Vietnam may be due to prevalence of viruses and co-infection. RSV was the most commonly isolated pathogen from China (31\%), followed by rhinovirus $(20 \%)$ and influenza virus $(13 \%)$. In contrast to this HRV was the most commonly isolated pathogen from Vietnam $(85 \cdot 3 \%)$. The number of cases with co-infection were also different in the two countries $13(14 \%)$ in China and two (3\%) in Vietnam. In multivariate analysis, we adjusted for country and type of virus. Limited data are available regarding the prevalent viruses circulating in China during the study period. For the trial 1 period, all influenza was influenza A(H1N1)pdm. For the trial 2 period, $21 \cdot 3 \%$ were $\mathrm{H} 1 \mathrm{~N} 1 \mathrm{pdm}, 2 \cdot 9 \%$ were $\mathrm{H} 3 \mathrm{~N} 2,3 \cdot 0 \%$ were influenza B Victoria, 2.6\% were influenza B Yamagata, $71 \cdot 2 \%$ were influenza A unsubtyped (Y. Zhang, Beijing Centre for Disease Prevention and Control, personal communication). We could not obtain data on the viruses circulating in Vietnam during the study period.

There are some limitations to this study. We did not subtype the influenza strains, and studies show that the rate of fever also varies between influenza strains [7-10, 18]. Fever data was self-reported but self-measured in three trials using a traditional glass and mercury thermometer. Lower fever rates in Chinese HCWs in this study might be due to due to differences in circulating viruses (and their pyrogenicity) between the two countries when the studies were conducted. A Japanese study of children with influenza reported a tendency towards shorter duration of fever with increasing age in children [18]; however, age and other demographic characteristics were not significant in that study.

\section{CONCLUSION}

Compared to children, this study shows that adults are less likely to have fever with a respiratory viral infection, even influenza. The implication of this finding is that for rapid treatment and reducing the risk of transmission of infection, clinicians should be aware that a diagnosis of viral respiratory infection, even influenza, is possible in the absence of fever. Many of these infections are transmissible even when infected persons are asymptomatic or presymptomatic, and greater vigilance for respiratory symptoms in HCWs could reduce nosocomial transmission of respiratory viral infections. The absence of fever should not preclude a differential diagnosis of influenza or other respiratory viruses in adults.

\section{ACKNOWLEDGEMENTS}

The authors thank the staff at the Beijing Centre for Disease Control and National Institute of Hygiene and Epidemiology. Thanks are also due to the staff from the hospitals in China and Vietnam which participated. $3 \mathrm{M}$ helped in fit testing during the first RCT in China, no financial support was provided. The second RCT in China was funded through the Australian National Health \& Medical Research Council of Australia (grant no. 630787). Funding to conduct the Vietnam trial study was received from the Australian Research Council (ARC) (grant no. LP0990749).

\section{DECLARATION OF INTEREST}

Dr Abrar Chughtai had testing of filtration of masks by $3 \mathrm{M}$ for his $\mathrm{PhD}$. Professor C. Raina MacIntyre has held an Australian Research Council Linkage Grant with $3 \mathrm{M}$ as the industry partner, for investigator-driven research. 3M have also contributed supplies of masks and respirators for investigator-driven clinical trials. She has received research grants and laboratory testing as in-kind support from Pfizer, GSK and Bio-CSL for investigator-driven research. The remaining authors have no competing interests to declare.

\section{REFERENCES}

1. Long CE, et al. Influenza surveillance in communitydwelling elderly compared with children. Archives of Family Medicine 1997; 6: 459-465.

2. Falsey AR, et al. Viral respiratory infections in the institutionalized elderly: clinical and epidemiologic findings. Journal of the American Geriatrics Society 1992; 40: 115-119.

3. Falsey AR, et al. Respiratory syncytial virus and influenza A infections in the hospitalized elderly. Journal of Infectious Diseases 1995; 172: 389-394. 
4. MacIntyre CR, et al. Respiratory viruses transmission from children to adults within a household. Vaccine 2012; 30: 3009-3014.

5. Ridgway JP, et al. Influenza among afebrile and vaccinated healthcare workers. Clinical Infectious Diseases 2015; 60: 1591-1595.

6. Cate TR. Clinical manifestations and consequences of influenza. American Journal of Medicine 1987; 82: 15-19.

7. Monto AS, Koopman JS, Longini IM. Jr. Tecumseh study of illness. XIII. Influenza infection and disease, 1976-1981. American Journal of Epidemiology 1985; 121: 811-822.

8. Wright PF, Thompson J, Karzon DT. Differing virulence of $\mathrm{H} 1 \mathrm{~N} 1$ and $\mathrm{H} 3 \mathrm{~N} 2$ influenza strains. American Journal of Epidemiology 1980; 112: 814-819.

9. Peltola V, Ziegler T, Ruuskanen O. Influenza A and B virus infections in children. Clinical Infectious Diseases 2003; 36: 299-305.

10. Kaji M, Watanabe A, Aizawa H. Differences in clinical features between influenza A H1N1, A H3N2, and B in adult patients. Respirology (Carlton, Vic) 2003; 8: 231-233.

11. Harper SA, et al. Seasonal influenza in adults and children - diagnosis, treatment, chemoprophylaxis, and institutional outbreak management: clinical practice guidelines of the Infectious Diseases Society of America. Clinical Infectious Diseases 2009; 48: 1003-1032.

12. Heinonen $\mathbf{S}$, et al. Signs and symptoms predicting influenza in children: a matched case-control analysis of prospectively collected clinical data. European Journal of Clinical Microbiology \& Infectious Diseases 2012; 31: 1569-1574.

13. Monto AS, et al. Clinical signs and symptoms predicting influenza infection. Archives of Internal Medicine 2000; 160: 3243-3247.

14. Lau LL, et al. Viral shedding and clinical illness in naturally acquired influenza virus infections. Journal of Infectious Diseases 2010; 201: 1509-1516.

15. Babcock HM, Merz LR, Fraser VJ. Is influenza an influenza-like illness? Clinical presentation of influenza in hospitalized patients. Infection Control and Hospital Epidemiology 2006; 27: 266-270.

16. Norman DC. Fever in the elderly. Clinical Infectious Diseases 2000; 31: 148-151.

17. Macintyre CR, et al. Ischaemic heart disease, influenza and influenza vaccination: a prospective case control study. Heart 2013; 99: 1843-1848.

18. Suzuki E, Ichihara K, Johnson AM. Natural course of fever during influenza virus infection in children. Clinical Pediatrics 2007; 46: 76-79.

19. Cao B, et al. Clinical features of the initial cases of 2009 pandemic influenza A (H1N1) virus infection in China. New England Journal of Medicine 2009; 361: 2507-2517.

20. Dai XQ, et al. Clinical predictors for diagnosing pandemic (H1N1) 2009 and seasonal influenza (H3N2) in fever clinics in Beijing, China. Biomedical and Environmental Sciences 2012; 25: 61-68.

21. MacIntyre CR, et al. A cluster randomised trial of cloth masks compared to medical masks in healthcare workers. BMJ Open 2015; 5: e006577.
22. MacIntyre CR, et al. A cluster randomized clinical trial comparing fit-tested and non-fit-tested N95 respirators to medical masks to prevent respiratory virus infection in health care workers. Influenza and Other Respiratory Viruses 2011; 5: 170-179.

23. MacIntyre CR, et al. A randomized clinical trial of three options for N95 respirators and medical masks in health workers. American Journal of Respiratory and Critical Care Medicine 2013; 187: 960-966.

24. Rodriguez-Noriega E, et al. Hospital triage system for adult patients using an influenza-like illness scoring system during the 2009 pandemic-Mexico. PLoS ONE 2010; 5: e10658.

25. Travers D, Gilboy N, Rosenau A. Pandemic Influenza Triage Tools: User Guide. Centers for Disease Control and Prevention, Healthcare Preparedness Activity (CDCHPA) and Oak Ridge Institute for Science and Education (ORISE).

26. Boivin G, et al. Predicting influenza infections during epidemics with use of a clinical case definition. Clinical Infectious Diseases 2000; 31: 1166-1169.

27. Carrat F, et al. Evaluation of clinical case definitions of influenza: detailed investigation of patients during the 1995-1996 epidemic in France. Clinical Infectious Diseases 1999; 28: 283-290.

28. Fox JP, et al. Influenzavirus infections in Seattle families, 1975-1979. I. Study design, methods and the occurrence of infections by time and age. American Journal of Epidemiology 1982; 116: 212-227.

29. Patrozou E, Mermel LA. Does influenza transmission occur from asymptomatic infection or prior to symptom onset? Public Health Reports (Washington, DC) 2009; 124: 193-196.

30. Putto A, Ruuskanen O, Meurman O. Fever in respiratory virus infections. American Journal of Diseases of Children 1986; 140: 1159-1163.

31. van den Hoogen BG, et al. Prevalence and clinical symptoms of human metapneumovirus infection in hospitalized patients. Journal of Infectious Diseases 2003; 188: 1571-7.

32. Peiris JS, et al. Children with respiratory disease associated with metapneumovirus in Hong Kong. Emerging Infectious Diseases 2003; 9: 628-633.

33. Manoha C, et al. Epidemiological and clinical features of hMPV, RSV and RVs infections in young children. Journal of Clinical Virology 2007; 38: 221-226.

34. Aberle $\mathbf{J H}$, et al. Single versus dual respiratory virus infections in hospitalized infants: impact on clinical course of disease and interferon-gamma response. Pediatric Infectious Disease Journal 2005; 24: 605-610.

35. Franz A, et al. Correlation of viral load of respiratory pathogens and co-infections with disease severity in children hospitalized for lower respiratory tract infection. Journal of Clinical Virology 2010; 48: 239-245.

36. Goka EA, et al. Single, dual and multiple respiratory virus infections and risk of hospitalization and mortality. Epidemiology and Infection 2015; 143: 37-47.

37. Drews AL, et al. Dual respiratory virus infections. Clinical Infectious Diseases 1997; 25: 1421-1429. 
38. Calvo C, et al. Multiple simultaneous viral infections in infants with acute respiratory tract infections in Spain. Journal of Clinical Virology 2008; 42: 268-272.

39. Martin ET, et al. Multiple versus single virus respiratory infections: viral load and clinical disease severity in hospitalized children. Influenza and Other Respiratory Viruses 2012; 6: 71-77.

40. Arcavi L, Benowitz NL. Cigarette smoking and infection. Archives of Internal Medicine 2004; 164: 22062216.

41. Huttunen R, Heikkinen T, Syrjanen J. Smoking and the outcome of infection. Journal of Internal Medicine 2011; 269: $258-269$.
42. Vassallo R, et al. Cigarette smoke extract suppresses human dendritic cell function leading to preferential induction of Th-2 priming. Journal of Immunology (Baltimore, MD) 2005; 175: 2684-2691.

43. Mili F, et al. The associations of race, cigarette smoking, and smoking cessation to measures of the immune system in middle-aged men. Clinical Immunology and Immunopathology 1991; 59: 187-200.

44. Gerna G, et al. Correlation of rhinovirus load in the respiratory tract and clinical symptoms in hospitalized immunocompetent and immunocompromised patients. Journal of Medical Virology 2009; 81: 14981507. 